\title{
Reprodutibilidade de um questionário de atividade física em escolares de 9 a 15 anos de idade
}

\author{
Reproducibility of a questionnaire on physical activity \\ among school students from 9 to 15 years of age
}

\author{
Eliane Denise Araújo Bacil ${ }^{1}$ \\ Thiago Silva Piola ${ }^{1}$ \\ Priscila Iumi Watanabe ${ }^{1}$ \\ Michael Pereira da Silva ${ }^{1}$ \\ Rosimeide Francisco Santos Legnani ${ }^{1}$ \\ Wagner de Campos ${ }^{1}$
}

${ }^{1}$ Departamento de Educação Física,

Universidade Federal do

Paraná. R. Coração de Maria

92, Jardim Botânico. 80215-

370 Curitiba PR Brasil.

elianebacil@hotmail.com
Abstract The objective of this study was to analyze the "test-retest" reproducibility of a questionnaire on physical activity among 1189 school students of both genders, from 9 to 15 years age in public schools in Curitiba/PR. The reproducibility of the questionnaire was determined by the repeated measures procedure called "test-retest", with an interval of one week between applications. For data analysis the Intraclass Correlation Coefficient (ICC), the percentage of correlation, the Kappa index, adjusted Kappa (Kappa PA$B A K)$ and Bland-Altman scatter diagram were used. Statistical analyses were performed using SPSS 21.0 adopting a 5\% significance level. All ICC were higher than 0.80. The correlation between the application replicas of the questionnaire was moderate, Kappa ranging from 0.51 to 0.61. There was a correlation between the questionnaire applications, the average difference between the first and second being equal to $106.49 \mathrm{~min} / \mathrm{wk}$ (IC95\%: 79.05-133.92) with limits of correlation ranging from 1070.97 (+2SD) to -857.99 (-2SD) min/week. In conclusion, the survey analyzed in this study showed satisfactory levels of reproducibility, having the possibility of being used to measure physical activity in both children and adolescents.

Key words Reproducibility of results, Questionnaire, Physical activity, Students
Resumo O objetivo deste estudo foi analisar a reprodutibilidade "teste-reteste" de um questionário de Atividade Física em 1.189 escolares, de ambos os sexos, de 9 a 15 anos da rede pública de ensino de Curitiba/PR. A reprodutibilidade do questionário foi determinada pelo procedimento de medidas repetidas "teste-reteste" com uma semana de intervalo entre as aplicações. Para a análise dos dados utilizou-se o Coeficiente de Correlação Intraclasse (CCI), o percentual de concordância, o indice Kappa, o Kappa ajustado (Kappa PABAK) e o diagrama de dispersão de Bland-Altman. As análises estatísticas foram efetuadas no SPSS 21.0 adotando-se um nível de significância de 5\%. Todos os CCI foram superiores a 0,80. A concordância entre as réplicas de aplicação do questionário foi moderada, Kappa variando de 0,51 a 0,61 . Verificou-se concordância entre as réplicas de aplicação do questionário, sendo a diferença média entre a primeira e a segunda igual a 106,49 min/semana (IC95\%: 79,05-133,92) com limites de concordância variando de 1.070,97 (+2DP) a -857,99 (-2DP) min/semana. Conclui-se que o questionário analisado neste estudo apresentou niveis satisfatórios de reprodutibilidade podendo ser utilizado para mensurar a atividade física tanto em crianças quanto em adolescentes.

Palavras-chave Reprodutibilidade dos resultados, Questionário, Atividade física, Estudantes 


\section{Introdução}

A atividade física (AF) apresenta-se como um importante comportamento relacionado a manutenção da saúde, independente da faixa etá$\mathrm{ria}^{1}$. Sua prática insuficiente tem sido identificada como um dos maiores problemas de saúde pública do século $21^{2}$. Estima-se que a inatividade física seja responsável por cerca de $9 \%$ de mortes prematuras em todo o mundo ${ }^{3}$. Tais dados ressaltam a característica pandêmica deste comportamento sendo este, a quarta principal causa de morte no mundo ${ }^{4}$.

A infância e a adolescência apresentam-se como um importante período de aquisição de comportamentos que tendem a manter-se na idade adulta ${ }^{5}$. Em relação a AF, dados apontam um tracking deste comportamento ao longo dos anos com declínio substancial durante a transição da adolescência para a idade adulta ${ }^{6-8}$. Verifica-se também que quanto maior a participação em diversos tipos de atividades físicas durante esta fase, maiores as chances de manutenção de níveis adequados de prática de AF no lazer na idade adulta ${ }^{6}$.

A avaliação da AF apresenta-se como um importante fator de monitoramento de saúde, visto a relação protetora de sua prática suficiente com doenças crônico degenerativas e mortalidade ${ }^{3}$. Diversos são os métodos e técnicas para mensurar a AF9. Embora existam instrumentos que permitam sua avaliação objetiva, os questionários são os instrumentos mais utilizados devido ao baixo custo, a fácil aplicação, a boa aceitação dos participantes e a rapidez na obtenção dos dados ${ }^{10-12}$ favorecendo sua utilização em estudos de larga escala visando a identificação de padrões de $\mathrm{AF}$ em estudos populacionais.

Ao se avaliar comportamentos relacionados a saúde é de suma importância a utilização de instrumentos de avaliação com propriedades psicométricas adequadas ${ }^{9-11}$. A reprodutibilidade teste-reteste é uma medida psicométrica que expressa a capacidade de um instrumento produzir os mesmos resultados ou resultados semelhantes entre réplicas de aplicação do instrumento, no mesmo grupo de pessoas, sob as mesmas condições e de forma independente refletindo a estabilidade temporal da medida ${ }^{13}$. Contudo, um determinado questionário pode apresentar níveis satisfatórios de reprodutibilidade em uma determinada população, mas não terá necessariamente o mesmo resultado quando aplicado em outras populações ${ }^{14}$. Podendo, tais discrepâncias serem resultado de um viés "cultural" causado por características distintas entre as populações ${ }^{15}$.
O Self-Administered Physical Activity Checklist $^{16}$, em sua versão adaptada, apresenta-se como um questionário recordatório para a avaliação da AF realizada nos sete dias anteriores a sua aplicação. Farias Junior et al. ${ }^{17}$ verificaram suas características psicométricas em uma amostra de adolescentes de 14 a 19 anos do município de João Pessoa (PB), região Nordeste do Brasil, identificando níveis elevados de reprodutibilidade "teste-reteste" e moderados níveis de validade.

O presente instrumento, apresenta uma lista das principais atividades físicas condizentes com características culturais de crianças e adolescentes brasileiros. Diante disto, além da capacidade de obtenção do tempo total gasto em AF, têm-se a possibilidade de identificação do tipo de atividade realizada pelo respondente.

Com o intuito de verificar sua aplicabilidade em escolares mais novos (a partir de 9 anos de idade) da região Sul do país, faz-se necessário verificar as capacidades psicométricas deste instrumento, visto que as particularidades apresentadas por populações de diferentes localidades, como a influência do clima, características ambientais e socioculturais, bem como para a utilização deste questionário em outras faixas etárias, podem resultar em resultados diferentes dos apresentados pelo estudo original. Adicionalmente, caso as propriedades psicométricas sejam adequadas, pode-se assumir que o instrumento pode ser aplicado nesta população de condições distintas, favorecendo o uso mais amplo deste instrumento, bem como a comparação entre populações de diferentes regiões.

Desta forma, este estudo tem como objetivo verificar a reprodutibilidade "teste-reteste" de um questionário de $\mathrm{AF}$ em escolares de 9 a 15 anos de uma cidade de Curitiba-PR.

\section{Métodos}

O presente estudo trata-se de um levantamento transversal de caráter intencional para avaliar a reprodutibilidade de um questionário de $\mathrm{AF}$. Participaram do estudo sete escolas, sendo três estaduais e quatro municipais da rede pública de ensino de Curitiba/PR.

A coleta de dados foi efetuada nos meses de março a dezembro de 2014, por uma equipe treinada do Centro de Estudos de Atividade Física e Saúde (CEAFS) da Universidade Federal do Paraná, supervisionados pela pesquisadora principal. Duas turmas por ano de ensino foram selecionadas aleatoriamente para participação da 
coleta de dados, e todos os alunos de cada turma sorteada foram convidados a participar do estudo. Inicialmente, foi solicitado autorização das escolas para realização do estudo. No dia anterior a coleta de dados foi entregue aos alunos o Termo de Consentimento Livre e Esclarecido (TCLE) e o Termo de Assentimento Livre e Esclarecido (TALE). O TCLE destinou-se a obter autorização dos pais ou responsáveis para que seus filhos pudessem participar da pesquisa e foi entregue a todos os alunos das turmas que iriam participar do estudo. O TALE foi entregue para os adolescentes acima de 12 anos assinarem concordando em participar do estudo. Na data estipulada da coleta de dados, de posse destes documentos, os escolares responderam o questionário em sala de aula, durante o horário normal de aula, a partir de orientações previas do grupo de aplicação.

Participaram desta pesquisa somente indivíduos entre 9 e 15,9 anos, sendo excluídos os escolares com idades $<9,0$ anos e $>15,9$ anos. Foi considerada como recusa quando o escolar não apresentou o TCLE assinado pelos pais/responsáveis ou quando o escolar se negou a participar da coleta de dados e não assinou o TALE. A perda amostral foi definida quando o escolar preencheu o questionário incorretamente ou desistiu da participação no estudo.

Para caracterização da amostra estudada, os adolescentes responderam a questões sobre aspectos sociodemográficos (sexo, idade, trabalho, moradia, tipo de residência), econômicos (classe econômica, escolaridade do pai e da mãe) e de nível de AF. Devido à amplitude da faixa etária, a idade decimal dos escolares foi obtida e classificada em três faixas etárias ( 9 a 10 anos, 11 a 12 anos e 13 a 15 anos).

A determinação da classe econômica foi realizada através do Critério de Classificação Econômica Brasil - $\mathrm{CCEB}^{18}$, o qual contabiliza a quantidade de itens existentes na casa de cada aluno, o grau de instrução do chefe da família ou do responsável que o sustenta e a presença de serviço público (água encanada e rua pavimentada). Baseando-se no somatório dos escores obtidos através destas informações, os escolares foram classificados nas seguintes classes: A (a mais alta), $\mathrm{B}, \mathrm{C}, \mathrm{D} / \mathrm{E}$ (a mais baixa). A escolaridade do pai e da mãe foi agrupada em duas categorias $(\leq 8$ anos de estudo e $>8$ anos de estudo).

O questionário de atividade física analisado no presente estudo é uma adaptação do Self-Administered Physical Activity Checklist ${ }^{16}$. Neste estudo, 55 meninos e 70 meninas do 5 ano de quatro regiões dos Estados Unidos, relataram os minutos durante o dia anterior que eles gastavam com 21 atividades físicas comuns. Para validação do instrumento, os escolares usaram simultaneamente um acelerômetro (sensor de movimento) e um monitor de frequência cardíaca durante pelo menos 8 horas do dia anterior. A correlação de Pearson entre o questionário com o índice de frequência cardíaca foi de 0,57 ( $p<0,001)$ e 0,30 $(\mathrm{p}<0,001)$ com a pontuação do acelerômetro.

No entanto, o Questionário de Atividade Física para Adolescentes proposto por Farias Junior et al. ${ }^{17}$, é composto por uma lista de $24 \mathrm{AF}$ de intensidade moderada a vigorosa (>3METS), com a possibilidade do escolar acrescentar mais duas. No preenchimento do questionário os escolares informaram a frequência (dias/semana) e a duração (horas e minutos por dia) das AF praticadas na última semana, considerando apenas as AF realizadas no lazer. Na determinação do nível de AF considerou-se o somatório do produto do tempo despendido em cada uma das AF pelas respectivas frequências de prática. Foram considerados suficientemente ativos os escolares com prática de AF igual ou superior a 420 minutos/semana e insuficientemente ativos aqueles que se exercitaram por um tempo menor ${ }^{1}$. O questionário apresentou níveis elevados de reprodutibilidade (Coeficiente de Correlação Intraclasse: CCI $=0,88$; IC95\%: 0,84-0,91) e moderados níveis de validade (índice kappa: $\mathrm{k}=0,59$ ) podendo ser utilizado para mensurar o nível de AF em adolescentes de 14 a 19 anos do município de João Pessoa (PB), região Nordeste do Brasil.

A reprodutibilidade foi estimada por meio do procedimento de medidas repetidas, com intervalo de uma semana entre as aplicações, utilizando-se de procedimentos idênticos àqueles adotados na primeira aplicação. A segunda aplicação do questionário foi realizada no mesmo dia da semana da primeira aplicação. Os adolescentes que não compareceram no dia e hora agendados para o preenchimento da réplica do questionário não fizeram parte da amostra do estudo. Ou seja, somente participaram do estudo os indivíduos que responderam o questionário na primeira e segunda aplicação.

A normalidade dos dados contínuos de AF foi avaliada por meio do teste de Kolmogorov e Smirnov. Os dados contínuos do nível de AF (réplicas de aplicação do questionário) não apresentaram distribuição normal. Para contornar o problema da normalidade, efetuou-se a transformação logarítmica dos dados. Para descrição das variáveis estratificadas por faixa etária utilizou-se a distribuição de frequências absoluta e relativa. 
O Coeficiente de Correlação Intraclasse (CCI) foi utilizado para determinar a reprodutibilidade "teste-reteste" do questionário. As medidas de concordância entre as réplicas de aplicação para a medida de AF em duas categorias incluíram o percentual de concordância, o índice Kappa e o Kappa ajustado (Kappa PABAK). O diagrama de dispersão de Bland-Altman foi utilizado para verificar a concordância absoluta entre réplicas de aplicação do questionário. As análises estatísticas foram realizadas através do software SPSS versão 21.0 adotando-se um nível de significância de 5\%.

O estudo foi aprovado pelo Comitê de Ética em Pesquisa com Seres Humanos da Universidade Federal do Paraná, de acordo com a Resolução do Conselho Nacional de Saúde (CNS) 466/2012.

\section{Resultados}

Participaram da primeira aplicação do questionário 1393 escolares, destes 69 foram excluídos por terem $<9,0$ anos e $>15,9$ anos de idade, 18 preencheram o questionário incorretamente e 117 não estavam presentes na sala de aula na segunda aplicação do questionário. Desta forma, fizeram parte do estudo 1189 escolares de 9 a 15 anos, de ambos os sexos.

A Tabela 1 apresenta as características sociodemográficas, econômicas e de nível de AF distribuídos por faixa etária.

A Tabela 2 apresenta os resultados da análise de reprodutibilidade "teste-reteste". Todos os CCI foram superiores a 0,80 . A concordância entre as réplicas de aplicação do questionário para o nível de AF em duas categorias foi moderada, Kappa variando de 0,51 a 0,61 e Kappa PABAK variando de 0,56 a 0,63 .

A Figura 1 apresenta o diagrama de dispersão de Bland-Altman para a concordância absoluta entre as duas aplicações do questionário. A diferença média observada entre as duas aplicações do questionário foram de aproximadamente duas horas/semana, ou seja, em média 106,49 minutos/semana (IC95\%: 79,05-133,92), podendo variar entre $1070,97(+2 \mathrm{DP})$ a $-857,99$ (-2DP) minutos/semana.

\section{Discussão}

O questionário apresentou níveis elevados de reprodutibilidade "teste-reteste" podendo ser utilizado para mensurar a AF em escolares, de ambos os sexos, de 9 a 15 anos de idade. De acordo
Tabela 1. Características sociodemográficas, econômicas e de nível de AF distribuídos por faixa etária $(\mathrm{n}=1189)$.

\begin{tabular}{|c|c|c|c|c|c|c|}
\hline \multirow[t]{2}{*}{ Variável } & \multicolumn{2}{|c|}{9 a 10 anos } & \multicolumn{2}{|c|}{$\begin{array}{c}11 \text { a } 12 \\
\text { anos }\end{array}$} & \multicolumn{2}{|c|}{$\begin{array}{c}13 \text { a } 15 \\
\text { anos }\end{array}$} \\
\hline & $\mathbf{n}$ & $\%$ & $\mathbf{n}$ & $\%$ & $\mathbf{n}$ & $\%$ \\
\hline Todos & 329 & 27,7 & 365 & 30,7 & 495 & 41,6 \\
\hline \multicolumn{7}{|l|}{ Sexo } \\
\hline Masculino & 185 & 56,2 & 181 & 49,6 & 239 & 48,3 \\
\hline Feminino & 144 & 43,8 & 184 & 50,4 & 256 & 51,7 \\
\hline \multicolumn{7}{|l|}{ Trabalho } \\
\hline Sim & 0 & 0,00 & 11 & 3,0 & 45 & 9,1 \\
\hline Não & 329 & 100,0 & 352 & 97,0 & 448 & 90,9 \\
\hline \multicolumn{7}{|l|}{$\begin{array}{l}\text { Com quem } \\
\text { mora }\end{array}$} \\
\hline $\begin{array}{l}\text { Com pai e } \\
\text { mãe }\end{array}$ & 200 & 60,8 & 240 & 65,8 & 295 & 59,6 \\
\hline $\begin{array}{l}\text { Com pai ou } \\
\text { mãe }\end{array}$ & 109 & 33,1 & 95 & 26,0 & 173 & 34,9 \\
\hline Outros & 20 & 6,1 & 30 & 8,2 & 27 & 5,5 \\
\hline \multicolumn{7}{|l|}{ Tipo de residência } \\
\hline Casa/sobrado & 298 & 90,6 & 327 & 89,6 & 447 & 90,5 \\
\hline $\begin{array}{l}\text { Apartamento/ } \\
\text { outro }\end{array}$ & 31 & 9,4 & 38 & 10,4 & 47 & 9,5 \\
\hline \multicolumn{7}{|l|}{ Classe econômica } \\
\hline A & 35 & 10,6 & 57 & 15,6 & 66 & 13,3 \\
\hline B & 164 & 49,8 & 222 & 60,8 & 275 & 55,6 \\
\hline $\mathrm{C}$ & 124 & 37,7 & 82 & 22,5 & 148 & 29,9 \\
\hline $\mathrm{D} / \mathrm{E}$ & 6 & 1,8 & 4 & 1,1 & 6 & 1,2 \\
\hline \multicolumn{7}{|c|}{ Escolaridade do pai } \\
\hline $\begin{array}{l}\leq 8 \text { anos de } \\
\text { estudo }\end{array}$ & 169 & 51,4 & 124 & 34,0 & 199 & 40,2 \\
\hline $\begin{array}{l}>8 \text { anos de } \\
\text { estudo }\end{array}$ & 160 & 48,6 & 241 & 66,0 & 296 & 59,8 \\
\hline \multicolumn{7}{|c|}{ Escolaridade da mãe } \\
\hline $\begin{array}{l}\leq 8 \text { anos de } \\
\text { estudo }\end{array}$ & 137 & 41,6 & 108 & 29,6 & 168 & 33,9 \\
\hline $\begin{array}{l}>8 \text { anos de } \\
\text { estudo }\end{array}$ & 192 & 58,4 & 257 & 70,4 & 327 & 66,1 \\
\hline \multicolumn{7}{|c|}{ Nível de Atividade Física } \\
\hline $\begin{array}{l}<420 \mathrm{~min} / \\
\text { sem }\end{array}$ & 118 & 35,9 & 154 & 42,2 & 208 & 42,0 \\
\hline $\begin{array}{l}>420 \mathrm{~min} / \\
\text { sem }\end{array}$ & 211 & 64,1 & 211 & 57,8 & 287 & 58,0 \\
\hline
\end{tabular}

com Landis e Koch ${ }^{19}$, CCI menores do que 0,40 expressam baixa correlação, entre 0,40 e 0,75 expressam boa correlação e maiores que 0,75 expressam excelente correlação. Neste estudo, todos os CCI foram superiores a 0,80 . Estes resultados corroboram o estudo de Farias Junior et al. ${ }^{17} \mathrm{com}$ adolescentes de 14 a 19 anos, onde a reproduti- 
Tabela 2. Medidas de reprodutibilidade "teste-reteste" do questionário considerando a medida contínua de atividade física e em duas categorias $(\mathrm{n}=1189)$.

\begin{tabular}{llllrc}
\hline \multicolumn{1}{c}{ Variável } & CCI & IC95\% & \%C & Kappa & Kappa PABAK \\
\hline $\begin{array}{l}\text { Todos } \\
\text { Sexo }\end{array}$ & 0,86 & $0,84-0,88$ & 80,2 & 0,58 & 0,60 \\
$\quad$ Masculino & 0,85 & $0,83-0,88$ & 81,5 & 0,57 & 0,63 \\
$\quad$ Feminino & 0,86 & $0,83-0,88$ & 78,9 & 0,58 & 0,58 \\
Faixa etária & & & & \\
$\quad$ 9 e 10 anos & 0,83 & $0,79-0,86$ & 78,1 & 0,51 & 0,56 \\
$\quad 11$ e 12 anos & 0,86 & $0,83-0,89$ & 81,1 & 0,60 & 0,62 \\
13 a 15 anos & 0,88 & $0,86-0,90$ & 81,0 & 0,61 & 0,62 \\
\hline
\end{tabular}

CCI: coeficiente de correlação intraclasse. IC95\%: intervalo de confiança a 95\%. \%C: coeficiente de concordância.

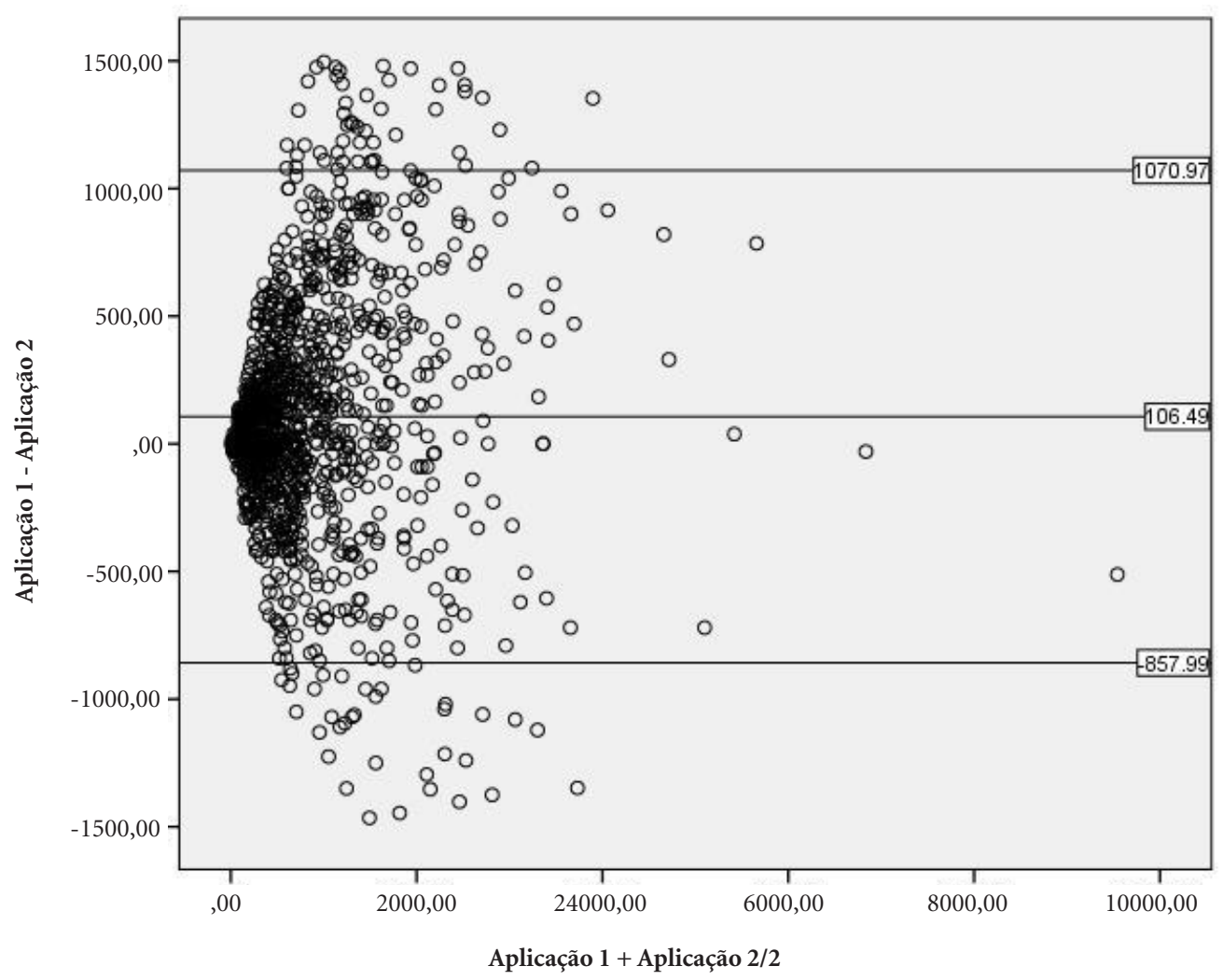

Figura 1. Gráfico de Bland-Altman para verificar o grau de concordância entre as réplicas de aplicação "testereteste" do questionário de atividade física.

bilidade "teste-reteste" foi elevada apresentando CCI = 0,88 (IC95\%: 0,84 - 0,91).

Tem-se observado uma grande variação nos coeficientes de reprodutibilidade "teste-reteste" dos questionários de AF de crianças e adolescentes. Farias Junior et al. ${ }^{9}$, realizaram uma revisão sistemática de estudos de reprodutibilidade e validade de instrumentos de medida da AF em adolescentes de 10 a 19 anos. A reprodutibilidade "teste-reteste" variou de 0,20 a 0,98 e a maioria dos coeficientes apresentou valores $<0,70$. Da mesma forma, Foley et al..$^{20}$ realizaram uma revi- 
são sistemática e encontraram CCI para reprodutibilidade variando de 0,24 a 0,98 .

Essas discrepâncias podem ser atribuídas às diferenças nas idades dos escolares que participaram dos estudos, bem como as diferenças no intervalo das aplicações do teste e do reteste. Intervalos prolongados entre as réplicas de aplicação do instrumento podem favorecer mudanças nas atividades praticadas resultando em subestimação da reprodutibilidade e intervalos muito curtos podem favorecer a superestimação dos resultados. Recomenda-se que o intervalo entre as aplicações do questionário seja curto, de um a três dias e não superior a uma semana ${ }^{21}$.

A concordância entre as réplicas de aplicação do questionário para o nível de $\mathrm{AF}$ em duas categorias foi moderada, com os coeficientes de Kappa variando de 0,51 a 0,61 e Kappa PABAK variando de 0,56 a 0,63 . O questionário de $A F$ avalia a última semana do escolar e não uma semana típica/normal. Desta forma, o comportamento do escolar pode ter sido diferenciado de uma semana para outra, o que pode justificar a concordância moderada encontrada entre as réplicas de aplicação do questionário. No entanto, estes resultados corroboram o estudo de Farias Junior et al. ${ }^{17} \mathrm{com}$ concordância moderada para medida de AF em duas categorias, Kappa variando de 0,42 a 0,58. Outros estudos apresentaram resultados semelhantes de concordância para medida de $\mathrm{AF}^{22-25}$.

A análise de Bland-Altman mostrou variabilidade na concordância das respostas de acordo com o tempo de AF semanal de cada indivíduo. Tais discrepâncias podem ter sofrido influência das características de medida do instrumento, visto que o mesmo avalia os últimos sete dias do participante. Sendo assim, a segunda aplicação não analisou o mesmo período de tempo da primeira, denotando uma provável alteração na rotina do escolar. Adicionalmente, as dificuldades em recordar algumas atividades podem influenciar esta variabilidade. No entanto, deve-se destacar que mesmo com a diminuição da consistência das respostas do tempo de AF, na maior parte dos casos, estes valores estavam dentro do intervalo de confiança de $95 \%$, bem como não houve excesso de valores discordantes de elevada magnitude e quase todas as diferenças individuais entre os pares de aplicação do questionário estavam dentro dos limites recomendados. Estes resultados foram semelhantes ao estudo de Farias Júnior et al. ${ }^{17}$, o qual apresentou o valor da diferença média de 118,1 (IC95\% 69,1-167,1) minutos/semana, com limites de concordância variando de $871,1(+2 \mathrm{DP})$ a $-639,4(-2 \mathrm{DP})$ minutos/semana. Resultados semelhantes foram encontrados nos estudos de Romero et al. ${ }^{22}$; Guedes et al. ${ }^{26}$; Guedes et al..$^{27}$ e Ridley et al. ${ }^{28}$.

Destaca-se como pontos positivos deste estudo o elevado tamanho amostral, a realização da pesquisa em escolas estaduais e municipais, bem como a possibilidade de utilização deste instrumento tanto em crianças quanto em adolescentes. Em relação às limitações, destaca-se a utilização de uma medida subjetiva de AF, o que pode ocasionar problemas de recordação e de estimação das informações que estão sendo mensuradas e a falta de validação concorrente com um instrumento padrão ouro como o uso do acelerômetro. Medidas subjetivas podem ser influenciadas por respostas que são socialmente aceitas ou esperadas pelos grupos de crianças e adolescentes. $\mathrm{Ou}$ tra limitação seria o fato de ser uma amostra intencional e não representativa da população de 9 a 15 anos de Curitiba/PR. Além disso, como neste estudo os escolares recordaram as AF praticadas em diferentes períodos (semanas distintas) nas duas aplicações, mudanças ocorridas nesse período podem ter contribuído para a subestimação das medidas de reprodutibilidade.

Conclui-se que o questionário apresentou concordância entre as réplicas de aplicação e níveis satisfatórios de reprodutibilidade, podendo ser utilizado para mensurar a AF tanto em crianças quanto em adolescentes. Sugere-se que novos estudos sejam realizados avaliando a validade concorrente do instrumento com a utilização da acelerometria. 


\section{Colaboradores}

EDA Bacil trabalhou na concepção e o delineamento ou a análise e interpretação dos dados, redação do artigo e sua revisão crítica, e na aprovação da versão a ser publicada. TS Piola, PI Watanabe, MP Silva e RFS Legnani trabalharam na redação do artigo e sua revisão crítica, e na aprovação da versão a ser publicada. W Campos trabalhou na concepção e o delineamento ou a análise e interpretação dos dados, redação do artigo e sua revisão crítica, e na aprovação da versão a ser publicada.

\section{Referências}

1. World Health Organization (WHO). Global recommendations on physical activity for health. Geneva: WHO; 2010.

2. Blair SN. Physical inactivity: the biggest public health problem of the 21st century. Br J Sports Med 2009; 43(1):1-2.

3. Lee IM, Shiroma EJ, Lobelo F, Puska P, Blair SN, Katzmarzyk PT. Effect of physical inactivity on major non-communicable diseases worldwide: an analysis of burden of disease and life expectancy. Lancet 2012; 380(9838):219-229.

4. Kohl HW, Craig CL, Lambert EV, Inoue S, Alkandari JR, Leetongin G, Kahlmeier S. The pandemic of physical inactivity: global action for public health. Lancet 2012; 380(9838):294-305.

5. Spring B, Moller AC, Coons MJ. Multiple health behaviours: overview and implications. Journal of public health (Oxford, England) 2012; 34(Supl. 1):i3-10.

6. Kjonniksen L, Torsheim T, Wold B. Tracking of leisure-time physical activity during adolescence and young adulthood: a 10-year longitudinal study. Int $J$ Behav Nutr Phys Act 2008; 5:69.

7. Telama R, Yang X. Decline of physical activity from youth to young adulthood in Finland. Med Sci Sports Exerc 2000; 32(9):1617-1622.

8. Telama R. Tracking of physical activity from childhood to adulthood: a review. Obesity facts 2009; 2(3):187195.

9. Farias Júnior JC, Lopes AS, Florindo AA, Hallal PC. Validade e reprodutibilidade dos instrumentos de medida da atividade física do tipo self-report em adolescentes: uma revisão sistemática. Cad Saude Publica 2010; 26(9):1669-1691.

10. Corder K, Ekelund U, Steele RM, Wareham NJ, Brage S. Assessment of physical activity in youth. J Appl Physiol (1985) 2008; 105(3):977-987.

11. Dollman J, Okely AD, Hardy L, Timperio A, Salmon J, Hills AP. A hitchhiker's guide to assessing young people's physical activity: Deciding what method to use. $J$ Sci Med Sport 2009; 12(5):518-525.

12. Bull FC, Maslin TS, Armstrong T. Global physical activity questionnaire (GPAQ): nine country reliability and validity study. J Phys Act Health 2009; 6(6):790-804.

13. Barros MVG, Reis RS, Hallal PC, Florindo AA, Farias Júnior JC. Análise de dados em saúde. Londrina: Midiograf; 2012.

14. Baranowski T. Validity and reliability of self report measures of physical activity: an information-processing perspective. Res Q Exerc Sport 1988; 59(4):314-327.

15. Shephard RJ. Limits to the measurement of habitual physical activity by questionnaires. Br J Sports Med 2003; 37(3):197-206.

16. Sallis JF, Strikmiller PK, Harsha DW, Feldman HA, Ehlinger S, Stone EJ, Williston J, Woods S. Validation of interviewer- and self-administered physical activity checklists for fifth grade students. Med Sci Sports Exerc 1996; 28(7):840-851. 
17. Farias Júnior JC, Lopes AS, Mota J, Santos MP, Ribeiro JC, Hallal PC. Validade e reprodutibilidade de um questionário para medida de atividade física em adolescentes: uma adaptação do Self-Administered Physical Activity Checklist. Rev Bras Epidemiol 2012; 15(1):198-210.

18. Associação Brasileira de Empresas de Pesquisa (ABEP). Critério de Classificação Econômica Brasil. 2015. [acessado 2016 Out 25]. Disponível em: http://www.abep. org/criterio-brasil.

19. Landis JR, Koch GG. The measurement of observer agreement for categorical data. Biometrics 1977; 33(1):159-174.

20. Foley L, Maddison R, Olds T, Ridley K. Self-report useof-time tools for the assessment of physical activity and sedentary behaviour in young people: systematic review. Obes Rev 2012; 13(8):711-722.

21. Hardy LL, Booth ML, Okely AD. The reliability of the Adolescent Sedentary Activity Questionnaire (ASAQ). Prev Med 2007; 45(1):71-74.

22. Romero A, Florindo AA, Voci SM, Slater B. Reprodutibilidade de questionário informatizado de atividade física em adolescentes. Rev Bras Ativ Fis Saude 2012; 16(3):234-239.

23. Booth ML, Okely AD, Chey TN, Bauman A. The reliability and validity of the Adolescent Physical Activity Recall Questionnaire. Med Sci Sports Exerc 2002; 34(12):1986-1995.

24. Verstraeten R, Lachat C, Ochoa-Aviles A, Hagstromer M, Huybregts L, Andrade S, Donoso S, Van Camp J, Maes L, Kolsteren P. Predictors of validity and reliability of a physical activity record in adolescents. $B M C$ Public Health 2013; 13(1109):1-11.

25. Philippaerts RM, Matton L, Wijndaele K, Balduck AL, De Bourdeaudhuij I, Lefevre J. Validity of a physical activity computer questionnaire in 12- to 18-year-old boys and girls. Int J Sports Med 2006; 27(2):131-136.

26. Guedes DP, Lopes CC, Guedes J. Reprodutibilidade e validade do Questionário Internacional de Atividade Física em adolescentes. Rev Bras Med Esporte 2005; 11(2):151-158.

27. Guedes DP, Lopes CC, Guedes J, Stanganelli LC. Reprodutibilidade e validade do questionário Baecke para avaliação da atividade física habitual em adolescentes. Rev Port Cien Desp 2006; 6(3):265-274.

28. Ridley K, Olds TS, Hill A. The Multimedia Activity Recall for Children and Adolescents (MARCA): development and evaluation. Int J Behav Nutr Phys Act 2006; 3(10):1-11.

Artigo apresentado em 21/03/2016

Aprovado em 11/11/2016

Versão final apresentada em 13/11/2016 